\title{
Social Competency, Sense of Loneliness and Self-Image among Reading Disabled (RD) Arab Adolescents
}

\author{
Eman Tarabia, Salim Abu-Rabia \\ Faculty of Education, University of Haifa, Haifa, Israel \\ Email: salimar@edu.haifa.ac.il
}

Received 26 February 2016; accepted 19 June 2016; published 22 June 2016

Copyright (C) 2016 by authors and Scientific Research Publishing Inc.

This work is licensed under the Creative Commons Attribution International License (CC BY).

http://creativecommons.org/licenses/by/4.0/

(c) (i) Open Access

\begin{abstract}
The aim of this study was to investigate social competency, sense of loneliness and self image among Arab dyslexic adolescent boys and girls. The study distinguished between boys and girls in the two groups, and examined the way dyslexia affects them, with the assumption that the Arab culture negatively affects the development of dyslexic girls more than that of dyslexic boys. The study was based on three main hypotheses which were tested by the quantitative research technique with the use of three questionnaires on which the study population replied: social competence questionnaire, sense of loneliness questionnaire and self-image questionnaire. In addition, two tests were held: A reading test and a reading comprehension test. The study population consisted of 87 Arab adolescent boys and girls: 19 dyslexic boys and 20 normal boys, 15 dyslexic girls and 33 normal girls. The study re-establishes and supports the assumption that the development of disability, the degree of disability and the difficulties are affected by the environment and culture of the disabled. Hence, the conservative, and traditional character of the Arab culture, collectively refers to girls differently than to boys, because it imposes on girls various prohibitions and restrictions from an early age, negatively affecting the personal, emotional and social development of Arab girls in overall, and that of girls with reading disability in particular. In the Arab culture a child with a learning disability has an inferior status, and his/her cognitive, emotional and social development is incompatible with that of his/her peers.
\end{abstract}

\section{Keywords}

Social Competency, Sense of Loneliness and Self-Image, Reading Disabled, Male and Female Arab Adolescents, Learning Disabilities and Arab Culture

\section{Introduction}

The aim of this study is to examine the characteristics of Arab students with learning disabilities (dyslexic) in 
adolescence, how these characteristics affect their academic achievements, and whether there is a difference between boys and girls. The study examines social characteristics-social competency and emotional characteristics-self-image and sense of loneliness, by questionnaires respectively.

In the past 20 years the literature which deals with children with learning disabilities, indicates a variety of social, emotional and behavioral difficulties, in addition to the academic difficulties they experience (Bryan, 1991; Bryan \& Bryan, 1981; Pearl, 1992). The study will test socio-emotional variables. In many studies, the social-emotional difficulties of individuals with learning disabilities were tested by using the variables: social competence and sense of loneliness (Lavian, 1996). The perception of social competence and the sense of loneliness are two sides of the same coin. Studies have shown that the loneliness variable has two main components, the emotional component and the social component (Anderson, Mullins, \& Johnson, 1987).

Various researchers attempted to examine in depth the effects of learning disability on the individual in terms of educational experiences. While many studies addressed LDs and their emotional and social aspects, still very few studies addressed LDs that look at adolescent boys and girls in the Arab sector.

Most studies are engaged in the developmental steps of primary school age and only few look at adolescence and the complexity of their development, especially in the Arab sector, whose conservative culture affects especially girls. Shulman (1995) states that the education in the traditional Arab culture and society, treats girls more strictly than boys, it imposes on girls more restrictions and prohibitions than on boys, such that require from them total obedience to all the cultural norms. In light of these characteristics it makes sense to look, at the current study, whether diagnosing reading disability in girls will adversely affect their emotional and social condition and will further intensify it, since they are also daughters of the Arab culture.

Reading is essential for successful functioning in society, and is the main means of acquiring knowledge and the basis for future employment. Most children learn to read effortlessly, but children with reading disability have difficulty in acquiring accurate and fluent reading. This difficulty causes them pain and frustration, and often prevents them from realizing their true intellectual ability. The possible causes and the characteristics that accompany reading disability, initiate an interest to study the phenomenon and its effects on the emotional and social aspect and on the achievements of the learner (Stanovich, 1986).

Margalit (1996) argued that the use of the multi-dimensional developmental model, has a theoretical and practical importance because conceptualization and understanding of the functioning of an individual suffering from learning disability, is contingent upon examining interactions which occur between the individual capabilities which are internal, and environmental variables. In other words, an examination of the capabilities in assignments and roles should be done in the context of age and culture presented to the individual, in different contexts and at different periods in his life. Thus, this study will attempt to contribute to the understanding of reading disabilities, and to a better understanding of the social and emotional characteristics experienced by disabled readers, especially adolescents of the Arab sector, while distinguishing between boys and girls in the cultural context. It is very important to check the Arab culture and environment affect on Arab girls who have reading disabilities more than boys, on the assumption that the patriarchal Arab culture is more supportive of boys than of girls. This understanding could lead to the construction of an appropriate intervention and teaching program that will assist students with reading disabilities. The purpose of this study, therefore, is to examine the existence of a possible link between reading disability, social competency, sense of loneliness, self-image and academic achievements (Margalit, 2014).

\section{Theoretical Background}

\subsection{Characteristics of Dyslexia}

Learning disability (L D) phenomena includes a number of major difficulties and disorders, and dyslexia or developmental reading disability, which is among the most common of them, is one of the most studied disabilities. It affects $80 \%$ of individuals who are defined as having learning disability and $15 \%$ to $10 \%$ of students in schools (Lyon, 1995; Vellution \& Fletcer, 2002). Reading is a complex cognitive task. Reading one sentence, requires several skills, from the identification of a single word to an understanding of the overall meaning of the text (Hoover \& Gough, 1990). Reading disability is expressed in non-fluent reading or inaccurate spelling of words (British Psychological Society, 1999).

The reading acquisition process is complex, and includes the development of phonological and orthographical skills, syntax and working memory. Normal readers develop these skills at age 9 - 7 years-the development of 
phonological skills facilitates the process of encoding words and their identification, and syntactic skills allow the reader to use the context and benefit from it while reading (Abu Rabia \& Siegel, 1995, Abu Rabia, 2002; Gough \&Tunmer, 1986; Siegel \& Ryan, 1984, 1988; Tunmer \& Chapman, 1998).

The International Association (National Joint Committee on Learning Disabilities-NJCLD) expanded and updated the 1994 definition of dyslexia, which stated that dyslexia is a specific learning disability of a neurobiological origin. It is characterized by difficulties in identification and precision of words and poor spelling and decoding abilities. These difficulties stem from impairment of the phonological component of the language, an impairment that does not necessarily due to the failure of other cognitive abilities and effective and satisfying classroom teaching. Secondary consequences may include problems in reading comprehension and reduced reading experience that can damage an increase in vocabulary and overall knowledge (Lyon, 1995; Shaywitz \& Shaywitz, 2004).

In Israel, dyslexia is defined as "a reading disability that is due to neuropsychological disruptions that harm the ability to achieve meaningful reading” (returns CEO in 1992). There are many factors for the phenomenon, and it is characterized by an inability to read at the level expected on the basis of age, intelligence and grade level, and there is a gap of at least two years between chronological age and the reading age (Margalit, 1996; Spector, 2005). Deficiencies in the ability to learn to read and reach fluency and accuracy in reading or derive information and meaning from written material can be due to a linguistic problem, impairment in deciphering writing which is expressed in omissions and/or addition of sounds or distortions and exchanges of sounds or parts of words. These defects are often referred to as “dyslexia” (CEO circular, 1996). Other researchers believe that dyslexia is a linguistic problem and some refer it to phonological difficulties which are formed in processing spoken language processes which affect phonological encoding speed and its accuracy (Farmer \& klein, 1995). In order to succeed in phonological and decoding and/or orthographic tasks, one requires a proper phonologicallexicon and an accurate representation of specific spelling models for words (Bernstein \& Tiegerman, 2008; Olson, 1994; Perfetti, 1994).

\subsection{Arab Society and LD Learners}

In the Arab society, the individual operates out of a need to avoid negative judgment of the environment, to maintain the dignity and good name and his family, keep away from "Shame" (Patai, 1973). Several studies examined the attitudes and perceptions about children with learning disabilities. These studies show that in the Arab society, attitudes toward Individuals with learning disabilities are negative (Mazzawi-Regina, 2001). It was also found that even in multicultural societies, Arabic speakers attitudes toward people with LD is more negative than those who speak other languages. It is important to note that these positions are not innate and are not universal but are acquired over a lifetime, and are part of the social norms and culture of the individual (Westbrook, Legge, \& Pennay, 1993; Zuniga, \& Fischer, 2010).

The present study examined the impact of dyslexia on adolescent boys and girls, thus it is appropriate to define adolescence and its characteristics.

\subsection{LD Adolescence}

Adolescence is a multi-turmoil age, which begins in early teens (12 - 10) and ends in the late teens (20 - 18). At this age, the adolescent undergoes physiological, emotional and social-familial changes requiring him to readjust. Since teenagers are not a uniform and homogeneous group, each adolescent at this age period, adapts differently (Moss, 1988). The main task facing the adolescent is to create a personal identity and to re-organize his/her personality, namely, move from childhood patterns to adulthood patterns. Identity develops by a dynamic and continuous interaction between the self and the other, out of an aspiration to sense belonging and form a positive self and group identity (Bandes-Jacob \& Friedman, 2000).

Adolescents with learning disabilities are characterized as having lower capacities than those of normal adolescents, in processes of visual memory and auditory-verbal memory. Also, based on self-reporting, they use less memory strategies than normal students. In the emotion sphere, it was found that during examinations, adolescents with learning disabilities get more excited than normal students and have more difficulty to regulate their emotions. Also, in the coping area, it was found that students with learning disabilities consider themselves less capable to cope than their normal peers (Ritter, 1989). Adolescents with learning disabilities feel the need to be accepted by the significant adults in their lives, not only by their parents but also by their teachers whom they 
see as their worthiness setters (Lavoie, 2005). In order to achieve a change in socio-emotional status, a dyslexic adolescent, needs support to assist him to advance and improve his emotions in order to allow him more social interactions, and more positive emotional and social experiences (Lev, 2008).

Since in adolescence, more than at a younger age, there is a marked difference between boys and girls, it is important to examine how this difference affects adolescent boys and girls with learning disabilities.

\subsection{Gender and LD}

It was found that the prevalence of depression symptoms and sense of loneliness are higher among adolescents than in children, and among girls more than in boys (Huntington \& Bender, 1993), due to the fact that adolescence is characterized by sharp changes in mood, hormonal changes, search for identity and the desire to achieve high social status (Adad, 2002; Moss, 1988). Azaiza and Ben Ari (1998), who examined the self-perception of Arab adolescents in Israel, indicated in their study, that Arab adolescent boys presented a more positive picture than that of Arab adolescent girls in regard to their emotions, their ability to control their own lives and the degree of satisfaction from their social lives. The girls introduced themselves as more nervous, easily hurt, anxious, tense and lonely.

Many studies that examined the source of the social difficulties of individuals with reading disablity, point to gender differences. According to Kandel and Davis (1986), social functioning of dyslexic girls will be deficient in their adulthood, due to them having emotional problems such as depression and anxiety. These emotional problems may cause them to be more introspective and interact less socially. That can lead them to a lack of social involvement, lack of social knowledge about how to behave in social situations, and an inability to interpret social and environmental cues. It was also found that adolescent boys with reading disability have social difficulties as a result of expressing more extrovert, aggressive and offensive behavior, than adolescent girls (Helms \& Gable, 1989). Girls reported of emotional problems leading to poor social functioning, while among boys there is no such report. The study of Tur-Kaspa and Bryan (1994) who examined the processing of social skills in dyslexic children found that dyslexic girls demonstrated significantly lower performance than dyslexic boys, namely, girls process social information less efficiently than boys, thus they interpret socialcues less well than boys.

The study of Gottieb, Gottieb and Levi (1986) found that dyslexic girls are more rejected and lonely and avoid forming social contractions than dyslexic boys. Settle and Milich (1999) examined social functioning by measuring social interaction failures, and found that dyslexic girls were seriously affected from a social interaction failure. Girls tended to talk less, were more passive and were less socially active. However, dyslexic adolescent boys, are less disciplined, more aggressive and impulsive. Battle (1992) claimed that dyslexic girls cooperate less with their peers than dyslexic boys. They exhibit more avoidable sensitive and distracted behavior than boys.

The difference between boys and girls in the social sphere requires investigating whether social capability is associated with social functioning in a disabled learner.

\subsection{Social Competency}

Social competency is the ability of the individual to create appropriate social relationships with others and develop them, those that would benefit him-self and others. Social Competence is defined as a learned behavior that affects the individual's relationship with peer adults group (Zuniga \& Fischer, 2010; Vaughn, 1985). This capability includes three function areas of the individual: (1) the behavioral domain-the capacity to initiate and sustain social interaction; (2) the emotional realm-the ability to express and regulate emotions; (3) the cognitive sphere-focuses on thinking and learning processes, how the individual perceives and interprets reality (Oliva-La Greca \& 1988).

Waters and Sroufe (1983) defined proper social behavior as the ability to use personal and environmental resources, to obtain personal or mutual gain in different spheres of life. This capacity allows achieving personal goals with social interactions in a socially acceptable manner, in order to maintain positive relations with others over time and in different situations. Hence, being a socially competent person is something based on the opinions of the people who are interacting with him, and it indicates that his social performance is compatible with what's accepted in his social circle, although it is not necessarily perfect. Thus, social competence is a complex global structure of specific social skills, which are not an innate character traits, but behaviors that are learned 
over the years.

Poor social competence is being explained as a disability in cognitive processes and difficulties in processing and understanding social knowledge effectively, in interpersonal communication and emotional difficulties. Consequently, the child finds it difficult to effectively and correctly interpret social events, to focus attention on significant social cues, to understand social situations, to draw conclusions and solve problems, and finds it difficult to analyze wrong information from the environment and understand others (Neuberger \& Margalit, 1998). As a result, children with learning disabilities have less chances to develop normal social relationships and function within them efficiently, and that leads to emotional difficulties. They are described in the literature as frustrated, unmotivated, unstable, rebellious, depressive and aggressive, anxious, with poor self-image, low selfesteem and lonely children (Margalit, 2014; Asher \& Gabriel, 1989; Asher, Parkhurst, Hymell, \& Williams, 1990; Gresham \& Elliot, 1987; Gresham \& Reschly, 1987). Due to under-developed social skills, for solving problems, they will use aggression instead of negotiation. The study of Dorothy Crawford (in Lavoi, 2005) showed that adolescent with learning disabilities faces three major risk factors regarding criminal behavior: more involvement in crime, will be more at risk to be arrested because of his crimes, and imposition in court of more severe penalties than those imposed on his normal friends.

\subsection{Loneliness and LD}

Common to the different definitions of loneliness is the individual's subjective perception about his relationship with others. In children with learning disabilities, loneliness leads to social problems, in that reducing opportunities to socialize, harms their personal and cognitive resources (Lavian Elul, Raviv and Hertz-Lazarowit, 2003). A relationship between loneliness and social skills in students with learning disabilities, has been found, although they are intertwined. Social relations serve as an image, and reflect the relationship between children's perception of the environment and its characteristics, and their beliefs and perceptions about friendship and loneliness (Margalit, 2014; Margalit \& Tur-Caspa, 1998). Children with learning disabilities experience a variety of difficulties in social relations. Their social status is lower, they are more rejected, they demonstrate lower social skills, suffer more than normal from behavior problems. As a result they report of dissatisfaction in their interpersonal relationships, high levels of loneliness and depression, and even suicidal tendencies (Al-Yagon \& Margalit, 2013; Bryan, 1977; Margalit \& Al-Yagon, 2002). In a study of Sharonnie and Bergstein (2010) on loneliness, self-image and social preference in adolescents with learning disabilities, the results showed that students with learning disabilities, particularly boys, reported of significantly higher loneliness and lower self-image than their normal counterparts (Sharonnie \& Bergstein, 2010).

Many studies have shown that strong sense of loneliness worsens social-emotional problems in children with learning disabilities in comparison to normal children (Zahavi, 1992; Yagil, 2006; Lavian Elul, Raviv, \& HertzLazorowitz, 2003; Sharonnie \& Bergstein, 2010; Asher et al., 1990; Gresham \& Reschlly, 1987; Margalit, 1994; Margalit, 2004). Checking the social and emotional areas and the relationship between social competency, learning disabilities and abnormal functioning is the result of the assumption, that difficulties in the acquisition of learning skills, may cause difficulties in acquiring social skills because learning difficulties may be accompanied by socio-emotional deficits (Heiman, 2000).

\subsection{Self-Image and LD}

Self-image is an estimation that an individual develops and enforces about himself. An individual's self-image develops and changes over time, and is affected by the attitude that a person receives already in childhood from those who surround him, especially during adolescence, which is a significant developmental period (Saada, 2009). Self-image can be defined as a collection of links, some of them internal, such as the identity of the person the way he sees it, his satisfaction from himself and from his behavior, as well as external aspects, such as body image, moral image, familial and social (Friedman, 2007). Self-image is a personal judgment of value, which is reflected in the attitudes that the individual has about himself. Self-image is a basic concept of personality theories. It won many names without fundamental distinction, among which are the self-concept, self-description, self-acceptance, self-esteem, self-perception and more. According to Wylie (1961) there is a substantial overlap between the various concepts that describe the self. Self-concept refers to the specific perception of a person of himself and the behavior that characterizes him. Self-concept is acquired on the basis of social interaction which is a central factor in determining a person's behavior (Wylie, 1961). 
Self identity is one of the areas in which the individual relates to himself and all the things that belong to him, such as personality traits, social affiliation, attitudes, beliefs and opinions. The behavior is the primary factor that shapes the identity. Therefore, the development of self-concept and self-image of children with learning disabilities is complex, because it is affected by the low academic performance at school alongside the social difficulties. Literature on the subject shows that children with learning disabilities report of low academic selfconcept levels, especially if their achievements are compared to those of normal students. They trust less their academic ability and their capacity to succeed in school, report a concern about their difficulties to cope with academic tasks, and on having to invest efforts and hard work to achieve the level of normal students, hence their sense of stress (Lackaye et al., 2006).

The current research questions and hypotheses, were derived from the literature which deals in learning disabilities that was brought, and they are presented below.

\subsection{Research Question}

What are the social characteristics (social competency) and emotional characteristics (self image and loneliness) of dyslexic students of the Arab sector, and how these characteristics affect their academic achievements?

\subsection{Hypotheses}

1) We will find a relationship between reading disability (dyslexia), self-image, social competency and sense of loneliness. This entirety will negatively affect academic achievement of dyslexic female and male students from the Arab sector.

2) We will find a statistically significant relationship between dyslexia and gender, and social-emotional condition of the student, thus that dyslexic Arab adolescent boys and girls will report on lower socio-emotional values compared to normal Arab adolescent boys and girls. In addition, that dyslexic Arab girls will report on lower socio-emotional level than that dyslexic Arab adolescents boys.

3) We will find a statistically significant relationship between gender and dyslexia and academic level of the student, so that the achievements of dyslexic adolescent Arab girls will be lower than the achievements of adolescents dyslexic adolescent Arab boys, and that achievement of dyslexic Arab adolescent girls will be lower than those of normal Arab adolescent boys and girls.

4) The social competence variable (on its four factors: assertiveness, cooperation, self-control and empathy) will explain the grade in reading and reading comprehension score, thus that a positive correlation between reading and reading comprehension tests scores and the four factors of social competence, will be found.

\section{Method}

\subsection{Participants}

Consisted of 87 Arab adolescents comprised of 19 dyslexic boys and 20 normal boys, 15 dyslexic girls and 33 normal girls. The study group comprised of 34 boys and girls who were diagnosed with learning disabilitiesdyslexic boys and dyslexic girls, according to the criteria of the Ministry of Education and didactic and psychological tests. The control group consisted of 53 normal female and male students, who were used as a control group in order to compare between them and the experimental group and obtain reliable results. The study population lives in northern Israel. The socioeconomic status of the participants is fair to well, and they study in junior high school in regular teaching and integrated teaching frameworks.

\subsection{Tools}

The study used three questionnaires: self image questionnaire; social competency questionnaire; and loneliness questionnaire. In addition, participants were tested on two achievement tests: a reading test that tests reading fluency and accuracy and a reading comprehension test.

Social competence questionnaire (Gresham \& Elliott, 1990) (Alpha = 0.80): "social competency questionnaire" is written in Hebrew and translated, for the purpose of the study, by the researcher into Arabic and was adapted according to need. The questionnaire includes 39 items that describe behavior in various social situations which are rated on a three levels scale: 1—“never”; 2-“sometimes”; 3-“often”. The resulting score is 
the average of the rankings at any position. The higher the sum, the higher the level of social competence, and vice versa. The scores ranged from 39 to 108 for the entire questionnaire. The questionnaire has provided scores in four categories: collaboration, assertiveness, self-control and empathy.

Collaboration: This category contains 10 items about behaviors like helping others and compliance with instructions, for example: "I am acting in accordance with the teacher's instructions."

Assertiveness: a category that contains 10 items about initiating behavior, such as asking for help from others, self-presentation and response to peer pressure and insults. An example of an item, "I ask for help from adults when children try to push me or to beat me up."

Self-control: the self-control category also includes 10 items dealing with behaviors that are revealed in conflict situations, such as the appropriate response to a behavior and in situations that may require acting by accepted norms. An example of an item: "I control my feelings when someone is angry with me."

Empathy: The fourth category deals with behaviors that express concern and appreciation for the feelings of someone else and his views. It includes 10 items, e.g. "I'm trying to figure out how my friends feel when they are angry, nervous or sad."

The sense of loneliness questionnaire (Asher et al, 1990) (Alpha $=0.84)$ : This area was tested by using a questionnaire that was given to students-"what I feel" (questionnaire is written in Hebrew but was translated into Arabicand edited for the purpose of the research). The questionnaire includes 24 items that examine the four following points: (1) a sense of loneliness of the child: "I am lonely," "I feel alone at school" and the like; (2) perception of the social situation of the child: "It is easy for me to make new friends at school," "I have many friends in my class"; (3) the degree of fulfillment of the child social needs,: "I can find a friend when I need to," "When I need help in school have no children I can turn to" and (4) social competence of the child: "I work well with other children".

In addition, the questionnaire includes questions designed to blur the intention of the questionnaire and describe areas of interests and activities, for example: "I watch a lot of TV", "I like music a lot", "I like computers." The items are rated on the basis of the five grades Likert scale: from 1-“I have never felt like this" to 5-"I always feel that way." The grade includes the a average score of 16 items excluding distracters.

Self-image questionnaire: compiled by Galantz (1989) (Alpha = 0.87) named "he (she) is so...and I like that." The questionnaire is based on the testimony of the person about himself in comparison to others, and its aim is to study his self-image at various reference centers. The questionnaire consists of 38 items. The respondent needs to choose one of three options, that seems the most appropriate in comparing himself with the presented image: 1-“I am similar to him”; 2-“I am not so similar to him”; 3-“I am very similar to him.” This questionnaire was also translated into Arabic to adapted to the study. The questionnaire is divided into the six self-image variables: personal traits, self-image in the eyes of his friends, self-image in the eyes of his teachers, self-image in the eyes of his father, self-image in the eyes of his mother, his attitude to school.

Reading fluency and accuracy test) (Alpha $=0.88$ ): The test was compiled by the researcher under the guidance of a team of teachers who teach the Arabic language. It includes 100 words rated from easy to difficult. Easy words consist of two to three letters and one or two syllables, as opposed to difficult words which consist of more syllables, and include four letters or more, and are usually punctuated with a sign which signals doubling the letter. The words were taken from Arabic study booklets approved by the Ministry of Education for junior high. The test was given individually in a quite room at the school. Each student read the list of words, and the examiner marked the number of words that were read correctly, and the number of words that were read incorrectly.

Reading comprehension test) (Alpha $=0.81$ ): The test was created by the researcher under the guidance of a team of teachers of Arabic and has a section in Arabic (the section name is-نهايتةاسدشجاع ("End”), by Arye Amitz, and was taken from a website called_هيالاليربية_Come to the Arabic” (www.alarabeyya.com). The section is appropriate for the participants age and includes 16 multi-choice questions. The questions are designed to identify level of knowledge and context from the text. Each question is followed by four possible answers and the child who is being tested has to choose the correct answer. All the answers are in the text. The section is punctuated, consists of two pages, and includes pictures illustrating the story content.

\subsection{Procedure}

The research was performed at school after obtaining the necessary permits from the appropriate authorities, 
such as an approval from the Chief Scientist, from the school principal, and consent of parents and students to participate in the study. Selection of the experimental group was done according to the recommendation of the school educational counselor and with her assistance while strictly guarding complete privacy of the tested students.

Questionnaires of self-image, social competency and sense of loneliness were given individually to the participants in the study and the control groups. That enabled reading the questionnaires to the dyslexics students and ascertain the extent of their reading comprehension, and mark the chosen answer. The questionnaires were presented at the school by a remedial teacher regardless of time limit, to ensure that all the details of the questionnaires and tests are met. The achievement tests (a reading test and reading comprehension test) were done by all the participants one week latter. All questionnaires and tests were conducted at school under appropriate conditions, strictly respecting the privacy of each subject, in a quiet room, a supportive learning environment and serene learning atmosphere. Before entering therepliesin each and every test questionnaire, participants received appropriate explanations and instructions. The subjects volunteered to participate in the study and were give an explanation about its aim. They were told that they can stop the process if they are tired or dissatisfied. It was also made clear them that the questionnaires are anonymous and will be used for research purposes only.

\section{Results}

The findings section includes statistical analysis and consists of two sections and sub-chapters. The first presents summing indices for the dependent variables of all the possible combinations of the independent variables, and the second presents the findings of the statistical analysis to examine the three hypotheses of the study.

- The dyslexic girls have the lowest empathy $(\mathrm{M}=1.24, \mathrm{Md}=1.2)$, self-control $(\mathrm{M}=1.12, \mathrm{Md}=1.1)$, assertiveness $(\mathrm{M}=1.07, \mathrm{Md}=1.1)$, cooperation $(\mathrm{M}=1.19, \mathrm{Md}=1.22)$ and overall social competence- $(\mathrm{M}=$ $1.157, \mathrm{Md}=1.18)$ scores, lower than the other boys and girls. The low range of the confidence intervals values at a confidence level of 95\% the dyslexic girls average for empathy (1.22 - 1.29), self control (1.06 1.18), assertiveness (1.03 - 1.1), cooperation (1.03 - 1:27) and overall social competence-(1.11 - 1.19) indicate a low social competency (both overall and in each of the four factors of social competence) in dyslexic girls.

- The central tendency of indices values and the values of confidence intervals at a $-95 \%$ confidence level of the dyslexics boys average for empathy $(1.55-1.88, \mathrm{M}=1.7105, \mathrm{Md}=1.6)$, self-control $(1.48-1.98, \mathrm{M}=$ 1.7263, $\mathrm{Md}=1.6)$, assertiveness (1.42 - 1.72, $\mathrm{M}=1.57, \mathrm{Md}=1.4)$, cooperation $(1.58-1.9, \mathrm{M}=1.74, \mathrm{Md}=$ $1.77)$ and overall social competence $-(1.56-1.81, \mathrm{M}=1.69869, \mathrm{Md}=1.62)$ indicate an almost medium social competence (both overall and in each of the of social competence factors) in dyslexics boys.

- The central tendency of indices values and the values of confidence intervals at a 95\% confidence level of the normal boys average for empathy ( $2.44-2.62, \mathrm{M}=2.51$, $\mathrm{Md}=2.5)$, self-control $(2.15-2.39, \mathrm{M}=2.27$, $\mathrm{Md}=2: 25)$, assertiveness (2.22 - 2:45, $\mathrm{M}=2.33, \mathrm{Md}=2.3)$, cooperation $(2.42-2.62, \mathrm{M}=2.52, \mathrm{Md}=2.44)$ and overall social competence- $(2.34-2.47, \mathrm{M}=2.41, \mathrm{Md}=2.38)$, indicate of an almost moderate social competence (both overall and in each of the causes of social competence) in normal boys.

- The central tendency of indices values and the values of confidence intervals at a 95\% confidence level-of the normal girls average for empathy $(2.28-2.49, \mathrm{M}=2.4, \mathrm{Md}=2.39)$, self control $(2.09-2.33, \mathrm{M}=(2.21$, $\mathrm{Md}=2.2$, assertiveness (2.18 - 2:38, $\mathrm{M}=2.28, \mathrm{Md}=2.3)$, cooperation $(2.35-2: 57, \mathrm{M}=2.46, \mathrm{Md}=2.66)$ and overall social competence- $(2.25-2: 42, \mathrm{M}=2.33$, $\mathrm{Md}=2.33)$, indicate of medium social competence (both overall and in each of the social competence factors) among normal girls students.

The results of the calculations of indices of central tendency for the sense of loneliness variable in the two variables, gender and dyslexia, show that:

- Dyslexic girls experience the highest sense of loneliness, $(M=4.45, \mathrm{Md}=4.37)$. The result of the calculation of confidence interval $95 \%$ at a confidence level for the average sense of loneliness in dyslexic girls (4.28 - 4.61) shows that in the 95\% confidence limits value 4.6 is within the confidence interval. This leads to the conclusion that at a 0.05 significance level, dyslexic girls feel lonely almost all the time.

- Dyslexic boys feel lonely in some cases $(\mathrm{M}=3.447, \mathrm{Md}=3.5)$. The result of the calculation at95\% confidence interval $95 \%$ for the average sense of loneliness in dyslexic boys (3.33 - 3.56) shows that at a confidence level of $95 \%$, the value 3.5 is within the limits of the confidence interval. This leads to the conclusion that at a 0.05 significance level, in some cases dyslexic boys feel lonely. 
- There is a similarity up to an identity between central tendency values of sense of loneliness of normal boys $(\mathrm{M}=2.04, \mathrm{Md}=2.09)$ and the corresponding values of sense of loneliness of normal girls $(\mathrm{M}=2.02, \mathrm{Md}=$ 2). Both confidence intervals, at 95\% confidence level of sense of loneliness in normal boys (1.69 to 2.37) and sense of loneliness in normal girls (1.79 to 2.26) show that in a 95\% confidence level, the value 2 lies between the two limits of each confidence interval.

\subsection{Summing Indices and Confidence Intervals for Self-Image Variables}

The results of the calculations of central tendency indices for the self image variable in the two variables, gender and dyslexia:

As for the overall self-image overall there is a similarity between the central tendency indices values of normal boys $(\mathrm{M}=2.003, \mathrm{Md}=1.89)$ and the corresponding values of normal girls $(\mathrm{M}=2.02, \mathrm{Md}=1.97)$. Examination of confidence intervals at a 95\% confidence level of the overall self-image average in normal boys (1.75 2.26) and that of normal girls (1.81 - 2.23), shows that at a confidence level of $95 \%$ the value 2 lies between the confidence interval limits (limits of each of the two confidence intervals) above). We can infer that at a 0.05 significance level, the overall self-image of normal boys and normal girls is medium

- The central tendency indices of the dyslexic boys $(\mathrm{M}=1.45, \mathrm{Md}=1.36)$ are higher than the corresponding values of dyslexic girls $(\mathrm{M}=1.03, \mathrm{Md}=1)$. At $95 \%$ confidence intervals confidence the overall self-esteemaverage in dyslexic boys (1.29 to 1.61) and dyslexic girls ( 0.99 to 1.05 ), show that at $95 \%$ confidence level the value 1.5 lies between value confidence interval limits of the overall self-esteem average in dyslexic boys, and the value of 1 is within the limits of the confidence interval of the overall self-image average in dyslexic girls. That indicates of a low to medium overall self-esteem in dyslexic boys, and low self-esteem in dyslexic girls.

- The value of the variable of attitude at home versus attitude at school, in dyslexic girls is 1 only. Getting a single value for a variable eliminates the important characteristic of the variable, namely its variability. Therefore, in statistical analyzes in which there was a need to refer to this variable, it was treated separately and independently of the rest of the self-image variables, while segmenting to combinations of the two variables-gender and dyslexia (except the dyslexic girls combination).

- The scores of the dyslexic girls for the self image factors are: personal characteristics $(M=1.02, M d=1)$, the image in the eyes of friends $(M=103, M d=1)$, the image in the eyes of the teacher $(M=1.02, M d=1)$, the image in the eyes of the father $(M=1.09, M d=1)$ image in the eyes of the mother $(M=1.02, M d=1)$, attitude to school $(\mathrm{Md}=1$, attitude at home versus at school $(\mathrm{M}=1, \mathrm{Md}=1)$ and the overall self-image $(\mathrm{M}=$ 1.03 , Md =1). Those scores were far lower than those of the other boys and girls. By looking at the confidence intervals results at a $95 \%$ confidence level of the average for the dyslexic girls for personality characteristics (0.99 to 1.05), the image in the eyes of friends ( 0.98 to 1.08$)$, the image in the eyes of the teacher ( 0.97 to 1.06$)$, the image in the eyes the father ( 0.97 to 1.19$)$ image in the eyes of the mother $(0.97$ to 1.1$)$, attitude to school (0.98 to 1.05 ) and overall self-image ( 0.99 to 1.05 ) we see that at 0.05 a significance level, the average value of dyslexia girls' self-image is 1 for each of the seven self-image factors, and also that the value of self-image in the attitude of dyslexic girls at home versus at school is certainly 1 . This indicates low self-esteem of dyslexic girls, both in overall and for each of the seven factors of self-image.

- The conclusion which can be drawn from looking at the confidence intervals values at a $95 \%$ confidence level of the average for dyslexic boys self-image, in personality traits (1.27 - 1.54), the image in the eyes of boyfriend $(1.15$ - 1.66), the image in the eyes of the teacher (1.03 - 1.5), the image in the eyes Father $(1.47$ 2.04), the image in the eyes of the mother (1.49 - 1.97), attitude to school (1.09 to 1.62), attitude at home versus at school $(1.26$ - 1.79) and the overall self-image $(1.29-1.61)$ is that at a 0.05 significance level the average value of self-esteem of the personal characteristics, the image in the eyes of the friends, the image in the eyes of the teacher, the image in the eyes of the father, image in the eyes of the mother, the attitude to school, attitude at home versus at school, in dyslexics boys are 1.5, 1.6, 1.5, 2, 1.9, 1.7 and 1.6respectively. This indicates an almost medium self-image of dyslexic boys both of overall self image and for each of the self-image factors, except for the image in the eyes of the father, for which (at a 0.05 confidence level) dyslexic boys feel that the image in the eyes of his father is medium.

- By studying the rows of the confidence intervals for each of the self-image variables of all possible combinations of the two variables-gender and dyslexia, we can see that in addition to the overlap of confidence 
interval ranges of normal boys average and those of normal girls average, there is an overlap of the confidence interval self image average values in dyslexic girls ( 0.97 to 1.06$)$ and that of the dyslexic boys (1.03 to 1.5). There is also an overlap of the values of the confidence interval average of each of the self-image variables, except for the personal characteristics variable in dyslexic boys, and the same average in normal boys and normal girls.

\subsection{Summing Indices for the Achievement Variables}

The two variables that indicate the students achievements are the reading score (the number of correctly read words out of 100 words in the reading test), and the reading comprehension score (the number of correct answers out of 16 answers in the reading comprehension test). The results of the summing indices calculation give rise to the following conclusions:

- The proportion of the correct answers of dyslexic boys reading test $((\operatorname{Pr})=0.48)$, is almost equal to the proportion of the correct answers in their reading comprehension test $(\mathrm{Pr})=0.47)$; The proportion of correct answers in the normal boys reading test $((\operatorname{Pr})=0.76)$ was similar to the proportion of the correct answers in their reading comprehension test $(\mathrm{Pr})=0.68)$; The proportion of the correct answers of dyslexic girls reading test $((\mathrm{Pr})=0.248)$, is similar to the proportion of the correct answers in their reading comprehension test $(\mathrm{Pr})$ $=0.32)$. The proportion of correct answers in the normal girls reading test $((\operatorname{Pr})=0.693)$ was equal to the proportion of the correct answers in their reading comprehension test $(\mathrm{Pr})=0.693)$.

- The reading achievements of dyslexic girls $((\mathrm{Pr})=0.248, \mathrm{M}=24.8, \mathrm{Md}=22)$ are the lowest, in comparison tothe other students categories achievements in this test.

- The reading comprehension achievements of dyslexic girls $((\operatorname{Pr})=0.0 .32, \mathrm{M}=5.133, \mathrm{Md}=5)$ are lower than those of the other students categories in this test.

- There are differences between the normal boys achievements $(\mathrm{Pr})=0.76, \mathrm{M}=76.1, \mathrm{Md}=87.5)$ and normal girls achievements $((\mathrm{Pr})=(0.693, \mathrm{M}=69.3, \mathrm{Md}=60)$ in the reading test. $50 \%$ of the normal boys read correctly 87.5 words or more, while $50 \%$ of the normal girls read correctly at least 60 words. This indicates that reading skills of normal boys are higher than those of normal girls.

- Reading comprehension achievements of normal boys and normal girls were similar: $((\mathrm{Pr})=0.68, \mathrm{M}=10.9$, $\mathrm{Md}=12)$ vesus $((\mathrm{Pr})=0.695, \mathrm{M}=11: 12, \mathrm{Md}=12)$ respectively.

- There are differences between the achievement of dyslexic boys in the reading test $((\mathrm{Pr})=0.48, \mathrm{M}=48.42$, $\mathrm{Md}=50)$ ) and the achievement of dyslexic girls in that test $((\operatorname{Pr})=0.248, \mathrm{M}=24.8, \mathrm{Md}=22)) .50 \%$ of the dyslexic boys could read correctly 50 words or more, while $50 \%$ of the dyslexic girls could read correctly at least 22 words. This difference indicates that dyslexic boys master reading better than dyslexic girls.

- There are differences between the achievement of dyslexic boys in the reading comprehension test $(((\operatorname{Pr})=$ $0.47, \mathrm{M}=7.53, \mathrm{Md}=8))$ and the achievement of dyslexic girls in that test $(((\mathrm{Pr})=0.32, \mathrm{M}=5.33, \mathrm{Md}=5))$. $50 \%$ of the dyslexic boys replied correctly on eight questions (out of 16 questions) or more. In contrast, 50\% of the dyslexic girls answered correctly on at least 5questions (out of 16 questions). This indicates that dyslexic boys, the reading comprehension of dyslexic boys is better than that of dyslexic girls, although the reading comprehension achievement of both dyslexic boys and dyslexic girls are low.

\subsection{Testing Research Hypotheses}

The first research hypothesis assumed noticing a statistically significant relationship between gender and dyslexia and emotional-social state of the student, namely, that Arab dyslexic adolescent boys and girls, will report of socio-emotional characteristic slower than those of normal Arab adolescent boys and girls and that dyslexic Arab adolescent girls will report of socio-emotional characteristic slower than those of dyslexic Arab adolescent boys.

The examination process of the first research hypothesis was divided into two phases. The first phase examined the relationship between the two independent variables and the social competence variables, and the second phase examined the relationship between the two independent variables and the emotional aspect variables.

\subsection{Testing the Relationship between the Gender and Dyslexia Variables and the Empathy}

The results of the two-way analysis of variance test, in which the independent variables with fixed effects are 
gender and dyslexia and the dependent variable is the empathy variable to the power of -1 with variable least squares weighted, which is one over the squared predicted residuals absolute.

The analysis of variance test results showed that at a 0.05 significance level there is a relationship between the interaction of two the variables, gender and dyslexia, and the empathy variable to the -1 power (and directly between the social competence variable empathy) $(\mathrm{F}(1,83)=40.347, p \approx 0<0.05)$. This result necessitate an examination of the relationship between each of the independent variables and the empathy variable to the power of -1 , given the other independent variable. The following summarizes the results of the two tests.

- There is a statistically significant difference in the empathy variable to the power of -1 (and directly in the empathy variable) between dyslexic boys and normal boys ( $\mathrm{F}(1,83)=79.9, p \approx 0<0.05$.). This result, together with the results obtained from the sample show that empathy in dyslexic boys $(M=1.71)$ is lower than empathy among normal boys $(\mathrm{M}=2.52)$.

- There is a statistically significant difference in the empathy variable to the power of -1 (and directly in the empathy variable) between dyslexic girls and normal girls $(\mathrm{F}(1,83)=586.5, p \approx 0<0.05)$. This result, together with the sample results show that empathy in dyslexic girls $(M=1.25)$ is lower than necessitate among normal girls $\mathrm{x}(\mathrm{M}=2.39)$.

- There is a statistically significant difference in the empathy variable to the power of -1 (and directly the variable empathy) between dyslexic boys and dyslexic girls $(\mathrm{F}(1,83)=67.7, p \approx 0<0.05)$. This result, together with the results obtained from the sample show that empathy in dyslexic boys $(M=1.71)$ is higher than empathy in dyslexic girls $(\mathrm{M}=1.25)$.

- There is no statistically significant difference in the empathy variable to the power of -1 (and directly in empathy) between normal boys and normal girls $(\mathrm{F}(1,83)=3.54, p=0.063>0.05)$. These results obtained from the sample for the empathy variable among boys and girls supports the result obtained (similarity between the empathy average in normal girls $(M=2.39)$ and the empathy average in normal boys $M=2.52)$ ).

\subsection{Testing the Relationship between the Gender and Dyslexia Variables and the Assertiveness}

The results of the two-way analysis of variance test, in which the independent variables with the fixed effects are the gender variable and the dyslexia variable and the dependent variable is the assertiveness variable to the power of minus half with variable least squares weighted, which is one over the squared residuals predicted absolute.

The analysis of variance test results showed that at a 0.05 significance level there is a relationship between the interaction of two the variables, gender and dyslexia, and the assertiveness variable to the power of minus half (and directly between the assertiveness variable) $(\mathrm{F}(1,83)=40.347, p \approx 0<0.05)$. This result necessitates an examination of the relationship between each of the independent variables and the assertiveness variable to the power of minus half, given the other independent variable. The following summarizes the results of the two tests.

At a 0.05 significance level, it can be concluded that:

- There is a statistically significant difference in the assertiveness variable to the power of minus half between dyslexic boys and normal boys $(F(1,83)=63.21, p \approx 0<0.05)$. The sample results show that the degree of assertiveness among dyslexic boys $(\mathrm{M}=1.57)$ is lower than the degree of assertiveness among normal boys $(\mathrm{M}=2.33)$.

- There is a statistically significant difference in the assertiveness variable to the power of minus half between dyslexic girls and normal girls $(\mathrm{F}(1,83)=721.58, p \approx 0<0.05)$. The sample results showed that the degree of assertiveness among dyslexic girls $(\mathrm{M}=1.07)$ is lower than the assertiveness level of normal girls $(\mathrm{M}=$ 2.28).

- There is a statistically significant difference in the assertiveness variable to the power of minus half between dyslexic boys and dyslexic girls $(\mathrm{F}(1,83)=69.92, p \approx 0<0.05)$. The sample results suggest that the degree of assertiveness among dyslexic boys $(\mathrm{M}=1.57)$, is higher than the degree of assertiveness among dyslexic girls $(\mathrm{M}=1.07)$.

- There is no statistically significant difference in the assertiveness variable to the power of minus half (and directly the assertiveness) between normal boys and normal girls $(F(1,83)=0.686, p=0.41>0.05)$. The similarity of the assertiveness average in normal girls $(M=2.28)$ and the assertiveness average in boys $(M=$ 
2.33), supports this outcome.

\subsection{Testing the Relationship between the Gender and Dyslexia Variable and the Self-Control}

The results of the two-way analysis of variance test, in which the independent variables with the fixed effects are the gender variable and the dyslexia variable and the dependent variable is the self control variable to the power of minus half with variable least squares weighted, which is one over the squared residuals predicted absolute.

The analysis of variance test results showed that at a 0.05 significance level there is a relationship between the interaction of the two variables, gender and dyslexia, and the self control variable to the power of minus half (and directly between self control variable) $(\mathrm{F}(1,83)=40.347, p \approx 0<0.05)$. This result necessitates an examination of the relationship between each of the independent variables and the self control variable to the power of minus half, given the other independent variable. The following are the results of the two tests.

At a 0.05 significance level, it can be concluded that:

- There is a statistically significant difference in the self-control variable to the power of minus half between dyslexic boys and normal boys $(\mathrm{F}(1,83)=26.94, p \approx 0<0.05)$. The sample results showed that the degree of self-control of dyslexic boys $(\mathrm{M}=1.73)$ is lower than the degree of self-control of normal boys $(\mathrm{M}=$ (2.28).

- There is a statistically significant difference in the self-control variable to the power of minus half (and directly in the self-control variable) between dyslexic girls and normal girls $(\mathrm{F}(1,83)=324.69, p \approx 0<0.05)$. The results obtained from the sample show that the degree of self-control of dyslexic girls $(\mathrm{M}=1.12)$ is lower than the degree of self-control of normal girls $(\mathrm{M}=2.21)$.

- There is a statistically significant difference in the self-control variable to the power of minus half (and directly the self-control variable) between dyslexic boys and dyslexic girls $(\mathrm{F}(1,83)=54.87, p \approx 0<0.05)$. The degree of self-control of dyslexic boys $(M=1.73)$ is higher than the degree of self-control of dyslexic girls $(\mathrm{M}=1.12)$.

- There is no statistically significant difference in the self-control variable to the power of minus half between normal girls and normal boys $(\mathrm{F}(1,83)=0.801, p=0.699>0.05)$. The similarity of the self-control average of normal girls $(M=2.21)$ and the self control average of normal boys $(M=2.28)$ supports this outcome.

\subsection{Testing the Relationship between the Gender and Dyslexia Variables and the Cooperation}

The statistical test that was utilized to examine the relationship between the gender dyslexia variables and collaboration variable is the two-way analysis of variance test, in which the independent variables with the fixed effects are the gender variable and the dyslexia variable and the dependent variable is the cooperation variable.

The analysis of variance test results showed that at a 0.05 significance level there is a relationship between the interaction of two the variables, gender and dyslexia, and the cooperation variable $(\mathrm{F}(1,83)=16.726, p \approx 0<$ 0.05). This result necessitates an examination of the relationship between each of the independent variables and the cooperation variable, given the other independent variable. The following summarizes the results of the two tests.

At a 0.05 significance level, we can conclude the following:

- There is a statistically significant difference in the cooperation variable between dyslexic boys and normal boys $(\mathrm{F}(1,83)=81.09, p \approx 0<0.05)$. The extent of cooperation among dyslexic boys $(\mathrm{M}=1.74)$ is lower than the extent of cooperation among normal boys $(\mathrm{M}=2.5)$.

- There is a statistically significant difference in the cooperation variable between dyslexic girls and normal girls $(\mathrm{F}(1,83)=229.04, p \approx 0<0.05)$. The extent of cooperation among dyslexic girls $(\mathrm{M}=1.19)$ is lower than the extent of cooperation in normal girls $(\mathrm{M}=2.46)$.

- There is a statistically significant difference in the cooperation variable between dyslexic boys and dyslexic girls $(\mathrm{F}(1,83)=35.43, p \approx 0<0.05)$. The extent of cooperation among dyslexic boys $(\mathrm{M}=1.74)$, is higher than the extent of cooperation among dyslexic girls $(\mathrm{M}=1.19)$.

- There is no statistically significant difference in the cooperation variable between normal boys and normal girls $(\mathrm{F}(1,83)=0.636, p=0.429>0.05)$. The similarity of the cooperation average among normal girls $(\mathrm{M}$ $=2.46)$ and the cooperation average among normal boys $(\mathrm{M}=2.5)$ supports this outcome. 


\subsection{Testing the Relationship between the Gender and Dyslexia Variables and the Overall Social Competence}

The statistical test that was used to examine the relationship between the gender and dyslexia variables and the overall social competency variable was the two-way analysis of variance test, in which the independent variables are gender and dyslexia and the dependent variable is the overall social competency variable to the power of minus quarter. In addition we used the variable least squares weighted, which is one over the squared predicted residuals absolute.

The analysis of variance test results showed that at a 0.05 significance level there is a relationship between the interaction of the, gender and dyslexia variables, and the overall social competency variable to the power of minus quarter $(\mathrm{F}(1,83)=40.347, p \approx 0<0.05)$. To carry on with the analysis, we will move on to examine the relationship between each of the independent variables and the overall social competence variable, given the other independent variable The following summarizes the results of the two tests.

At a 0.05 significance level, we can conclude the following:

- There is a statistically significant difference in the overall social competence to the power of minus quarter (and directly the overall social aspect) between dyslexic boys and normal boys $(\mathrm{F}(1,83)=119.99, p \approx 0<$ 0.05). This result, together with the results obtained from the sample show that the overall social aspect among dyslexic boys $(M=1.607)$ is lower than the overall social aspect of normal boys $(M=2.409)$.

- There is a statistically significant difference in the overall social competence variable to the power of minus quarter (and directly the overall social aspect) between dyslexic and normal girls $(\mathrm{F}(1,83)=690.9, p \approx 0<$ $0.05)$. The degree of the overall social competence variable in dyslexic girls $(M=1.156)$, is lower than the overall social competence variable in normal girls $(\mathrm{M}=2.33)$.

- There is a statistically significant difference in the overall social competence variable to the power of minus quarter between dyslexic boys and dyslexic girls $(\mathrm{F}(1,83)=115.68, p \approx 0<0.05)$. The extent of the overall social competence of dyslexic boys $(M=1.607)$ was higher than that of the dyslexic girls $(M=1.156)$.

- There is no statistically significant difference in the overall social competency variable to the power of minus a quarter between normal boys and normal girls $(\mathrm{F}(1,83)=2.587, p=0.114>0.05)$. The similarity of the social competence aspect average of normal girls $(\mathrm{M}=2.33)$ and the corresponding average in normal boys $(M=2.409)$, supports this outcome.

The results of the examination of the first research hypothesis which deals with the social competence variables: empathy, assertiveness, self-control, cooperation and overall social competence, can be summarized as follows.

- Social competence of dyslexic boys is lower than that of normal boys.

- Social competence of dyslexic girls is lower than that of normal girls. The social competence of dyslexic girls is low, whereas the social competence of normal girls is medium to high.

- Social competence in dyslexic girls is lower than that of boys dyslexic.

- Social competence of normal boys is identical to the social competence of normal girls.

\subsection{Testing the Relationship between the Dyslexia and Gender Variables and the Emotional Aspect}

The examination of the first research hypothesis in regard to the emotional aspect variables was divided into two parts: the first examined the combined effect of the two independent variables on loneliness, and the second looked at the existence of a joint influence of the two independent variables: a) on each of the self-image variables and $b$ ) on the overall self-image variable.

\subsection{Testing the Relationship between the Gender and Dyslexia Variables and the Sense of Loneliness}

The examination of the relationship between the independent variables gender and dyslexia, and the sense of loneliness variable was done with the two-way analysis of variance test, in which the independent variables (with the fixed effects) are the gender variable and the dyslexia variable, and the dependent variable was the sense of loneliness variable. In addition to these variables, we used the variable least squares weighted which is one over the squared predicted residuals absolute. 
The analysis of variance test results showed that at a 0.05 significance level there is a relationship between the interaction of the gender and dyslexia variables, and the sense of loneliness variable $(\mathrm{F}(1,83)=19.802, p \approx 0<$ 0.05). This result necessitates an examination of the relationship of each independent variable and sense of loneliness variable, given the other independent variable.

At a 0.05 significance level, we can conclude the following:

- There is a statistically significant difference in the sense of loneliness between dyslexic boys of and normal boys $((\mathrm{F}(1,83)=12.51, p=0.0006<0.05$. The results obtained from the sample show that the sense of loneliness in dyslexic boys $(\mathrm{M}=3.447)$ is higher than the sense of loneliness of normal boys $(\mathrm{M}=2.04)$.

- There is a statistically significant difference in the sense of loneliness between dyslexic girls and normal girls $(\mathrm{F}(1,83)=38.94, p \approx 0<0.05)$. The sense of loneliness in dyslexic girls $(\mathrm{M}=4.45)$ is higher than the sense of loneliness of normal girls $(\mathrm{M}=2.02)$.

Below, the results of the analysis of variance model that examined the relationship between gender and sense of loneliness given the dyslexia variable:

- There is a statistically significant difference in the sense of loneliness between dyslexic boys and dyslexic girls $(\mathrm{F}(1,83)=5.39, p=0.022<0.05)$. The sense of loneliness in dyslexic boys $(\mathrm{M}=3.447)$ is lower than the sense of loneliness of dyslexic girls $(\mathrm{M}=4.45)$.

- There is no statistically significant difference in the sense of loneliness between normal boys and normal girls $((\mathrm{F}(1,83)=(0.0006, p=0.979>0.05)$. The similarity of the sense of loneliness average in normal girls $(M=2.02)$ and sense of loneliness average of normal boys $(M=2.04))$ indicates no difference in the sense of loneliness between normal boys and normal girls.

Based on the above analysis, we can conclude that:

- The sense of loneliness of dyslexic boys is higher than that of normal boys.

- The sense of loneliness of dyslexic girls is higher than that of normal girls. Normal girls rarely feel lonely while dyslexic girls feel lonely in most situations.

- The sense of loneliness among dyslexic girls is higher than among dyslexic boys.

- Normal girls and normal boys hardly feel lonely.

\subsection{Testing the Relationship between the Gender and Dyslexia Variables and the Seven Self Image}

As was already mentioned, the examination of the relationship between the gender and dyslexia variables and self image was divided into two: the one, looked at the relationship between the independent variables and each of the seven factors of self image, and the second examined the relationship between the independent variables and the overall self image variable.

The examination of the relationship between the two independent variables, gender and dyslexia, and the seven factors of self image, it was necessary to separate the variable "attitude at home versus attitude at school" from the other self image variables. Thus, here too, the statistical analysis was divided into two parts: the one, an examination of the relationship between the gender dyslexia variable (which refers only to the three combinations dyslexic boys, normal boys and normal girls) and the "attitude at home versus attitude at school" variable. The second part is devoted to the examination of the relationship between gender-dyslexia and the six self image variables: personal characteristics, self image in the eyes of friends, self image in the eyes of his teachers, self image in the eyes of his father, self image in the eyes of his mother and attitude to school.

The statistical analysis which examined the relationship between the gender-dyslexia variable and the "attitude at home versus attitude at school" variable was done with one-way analysis of variance test in which the dependent variable is "attitude at home versus attitude at school" variable and the independent variable with the fixed effect is the gender-dyslexia variable.

At a significance level $0.05(\mathrm{~F}(1,69)=1.323, p=0.273>0.05)$, the difference between the three variable averages "attitude at home versus at school" dyslexic boys $(\mathrm{M}=1.53)$, normal boys $(\mathrm{M}=1.82)$, and the normal girls $(M=1.82)$ was not statistically significant.

Obtaining an average for "attitude at home versus at school" variable in dyslexic girls $(M=1)$ that is lower than the corresponding averages in each of the three combinations: dyslexic boys dyslexic normal boys and normal girls, indicates that the image in attitude at home versus in school for dyslexic girls, is lower than the images of dyslexic boys, normal boys and normal girls. 
The statistical test that was utilized to examine the relationship between the independent variables gender and dyslexia and the self-image variables personal characteristics, self image in the eyes of friends, self image in the eyes of his teachers, self image in the eyes of his father, self image in the eyes of his mother and attitude to school was the Multivariate Multivariate Analysis of Variance (MANOVA). In this test the independent variables are gender and dyslexia, and the group of the dependent variables are personal characteristics, self image in the eyes of friends, self image in the eyes of his teachers, self image in the eyes of his father, self image in the eyes of his mother and attitude to school.

- There are no differences between the four levels of interaction, dyslexia * gender (dyslexic boys normal boys, dyslexic girls and normal girls) in the combination of the six self-image variables, personal characteristics, self image in the eyes of friends, self image in the eyes of his teachers, self image in the eyes of his father, self image in the eyes of his mother and attitude to school (Wilks $\Lambda=0.816, \mathrm{~F}(6,78)=2.097, p=$ $0.063>0.05)$. The meaning of the result is that the differences between the averages of the four possible interactions combinations obtained in each of the six self-image variables, are not statistically significant at a 0.05 significance level. The result that was obtained at this stage made it possible to test the significance of the relationship between the two levels of gender variables and the combination of the six image variables and significance, and the relationship between the two levels of dyslexia and the same combination. The results of these two tests are given below.

- There are no statistically significant differences between the two levels of the gender and the combination of the six self image variables, personal characteristics, self image in the eyes of friends, self image in the eyes of his teachers, self image in the eyes of his father, self image in the eyes of his mother and attitude to school (Wilks $\Lambda=0.86$, F $(6,78)=2.117, p=0.061>0.05)$ ).

There are statistically significant differences between the two dyslexia levels and the combination of the six self image variables, personal characteristics, self image in the eyes of friends, self image in the eyes of his teachers, self image in the eyes of his father, self image in the eyes of his mother and attitude to school (Wilks $\Lambda$ $=0.487, \mathrm{~F}(6,78)=13.697, p \approx 0<0.05)$ ). The significance of the result obtained is, that at least for one self-image factor (out of the six factors) the dyslexic students (boys and girls) average is different than the normal students (boys and girls) average. This result led to the stage of detecting the differences between dyslexic and normal students in each of the six factors. The statistical analysis method with which those differences were detected, was the Bootstrap Re-The following summarizes the results obtained by that analysis method.

- At a 0.05 significance level, $(P \approx 0<0.05)$ we may conclude that the personal characteristics image of the dyslexic pupils $(M=1.24)$ is lower than that of normal students $(M=2.003)$.

- At a 0.05 significance level, $(P \approx 0<0.05)$ it can be concluded that the image in the eyes of friends of dyslexic pupils $(M=1.24)$ is lower than that of normal students $(M=1.93)$.

- At a 0.05 significance level, $(P \approx 0<0.05)$ it can be concluded that the image in the eyes of teachers of dyslexic pupils $(M=1.16)$ is lower than that of normal students $(M=1.99)$.

- At a 0.05 significance level, $(P \approx 0<0.05)$ it can be concluded that the image in the eyes of their father of dyslexic pupils $(M=1.46)$ is lower than that in normal students $(M=2.1)$.

- At a 0.05 significance level, $(P \approx 0<0.05)$ it can be concluded that the image in the eyes of their mother of dyslexic pupils $(M=1.42)$ is lower than that of normal students $(M=2.15)$.

- At a 0.05 significance level, $(P \approx 0<0.05)$ it can be concluded that the attitude of the dyslexic pupil to school $(M=1.42)$ is lower than that of normal students $(M=2.15)$.

Results of the examination of the relationship between the gender and dyslexia variables and the seven self image factors can be summarized in the following manner:

- As for the six factors of self-image, personal characteristics, the image of the student in the eyes of friends, the image of the student in the eyes of his teachers, the image of the student in the eyes of his father, the image of the student in the eyes of his mother, and the image of the student in regard to his attitude to school, no relationship was found between the interaction of gender and dyslexia and each of these six factors at a 0.05 significance level. By the same level of significance, there was no relationship between gender and each of these six factors. But we did find a relationship between the dyslexia variable and each of these six factors at a 0.05 significance level. In other words, for each factor, it was found that the self image of a dyslexic student (boy or girl) is lower than the self-image of a normal student (boy or girl).

- As for the attitude of the student at home versus the attitude at school, it was found that the self image of dyslexic girls is the lowest; the self image of dyslexic boys, of normal boys and of normal girls is identical 
(for that factor).

\subsection{Testing the Relationship between the Gender and Dyslexia Variables and the Overall Self Image}

The statistical test that was used to examine the relationship between the gender and dyslexia variables and the overall self-image was the two-way analysis of variance test, in which the independent variables with fixed effects are gender and dyslexia and the dependent variable is the overall self image variable to the power of -3 .

The analysis of variance test results show that at a 0.05 significance level, there is a relationship between the interaction of the two variables, gender and dyslexia, and the self-image variable to the power of -3 (and directly between the overall self-image variable) $(\mathrm{F}(1,83)=64.593, p \approx 0<0.05)$. This result led to the checking of the relationship between each of the two independent variables and overall self-image variable, given the other independent variable

Below, a summary of the two tests and the detailed statistical analysis of these two tests.

At a 0.05 significance level, we can conclude the following.

- The overall self-image in dyslexic boys $(\mathrm{M}=1.45)$ is lower than the overall self-image of normal boys $(\mathrm{M}=$ 2.003), $(\mathrm{F}(1,83)=18.35, p \approx 0<0.05)$.

- The overall self-image among dyslexic girls $(\mathrm{M}=1.03)$ is lower than the overall self-image of normal girls $(\mathrm{M}=2.02),(\mathrm{F}(1,83)=244.92, p \approx 0<0.05)$.

- The overall self-image in dyslexic boys $(\mathrm{M}=1.45)$ is higher than the overall self-image of dyslexic girls (M $=1.03),(\mathrm{F}(1,83)=105.766, p \approx 0<0.05)$.

- There is no statistically significant difference between the overall self-image of normal boys $(\mathrm{M}=2.003)$ and overall self-image of normal girls $(\mathrm{M}=2.02), \mathrm{F}(1,83)=0.029, p=0.864>0.05)$.

Based on this statistical analysis and the results obtained from the primary analysis in section 4.3, as for the relationship between the gender and dyslexia variables and the overall self-image variable we can conclude that:

- The overall self-image of dyslexic boys is lower than that of normal boys.

- The overall self-image of dyslexic girls is lower than that of normal girls. Whereas the self-image of dyslexic girls is low, the self-image of normal girls is medium.

- The overall self-image of dyslexic girls is lower than that of dyslexic boys.

- The overall self-image of normal girls and normal boys is medium.

\subsection{Testing the Second Research Hypothesis}

The second research hypothesis assumed finding a statistically significant relationship between gender and dyslexia and academic level of the student, namely, that the achievements of Arab dyslexic adolescent girls will be lower than the achievements of Arab dyslexic adolescent boys and that achievement of dyslexic and adolescent boys and girls will be lower than the achievements of normal adolescent boys and girls.

The academic level variables are the reading and the reading comprehension test scores. Therefore, in order to examine the second research hypothesis we looked at:

- The relationship between gender and dyslexia and students' achievement in a reading test-the number of correctly read words out of 100 words.

- The relationship between gender and dyslexia and students' achievement in reading comprehension test-the number of correct replies on 16 questions

The statistical analysis was done by the Generalized Estimation Equation (GEE).

According to this model, the dependent variable is the number of correct answers, the intra-test variable is the type of score variable (reading score and reading comprehensions core), the independent variables are the gender and dyslexia variables.

At a 0.05 significance level, it can be concluded that there is a relationship between the interaction, score type, gender and dyslexia and the score $\left(\chi_{\text {Wald }}^{2}(2)=7.003, p=0.03<0.05\right)$. For further analysis we examined the relationship between gender and dyslexia and each of the two dependent variables-reading score and reading comprehension score.

To examine the relationship between gender and dyslexia and the score variable (the reading score or the reading comprehension score) we used the Generalized Linear Models. These models examine a statistical relationship between dependent variable and independent variables, so that the distribution of the dependent variable 
is not necessarily normal. As is well known, the distribution of the dependent variable for each of the above tests is binomial, therefore the appropriate generalized linear model is a generalized linear model for binomial distribution. It should be noted, that the relationship function that is used for the implementation of the statistical test in this case, is the logit function.

\subsection{Testing the Relationship between Gender and Dyslexia and the Reading Score}

The Generalized Linear Implementation Models revealed that at a 0.05 significance level there is a relationship between the interaction gender and dyslexia and the reading score $\left(\chi_{\text {wald }}^{2}(1)=50.043, p \approx 0<0.05\right)$. For further analysis we examined the relationship between one of the dependent variables and the reading score, given the other independent variable. The test results at a significance level 0.05 , revealed that:

- In dyslexic pupils, thereis a relationship between the gender variable and the reading score variable ( $\chi_{\text {Wald }}^{2}(1)=203.19, p \approx 0<0.05$ ). The cross ratio value for a dyslexic boy (versus dyslexic girl) is $\mathrm{OR}=$ 2.847. That means that a dyslexic boy is inclined to read correctly Arabic words 2.847 times more than a dyslexic girl. The significance of that result is that the proportion of correctly read words, out of 100, by a dyslexic boy $((\mathrm{Pr})=0.48)$ is higher than the proportion of correctly read word by a dyslexic girl $((\mathrm{Pr})=$ 0.248 ).

- There is a relationship between the gender variable and the reading score variable in normal students $\left(\chi_{\text {Wald }}^{2}(1)=28.864, p \approx 0<0.05\right.$ ) . The cross ratio value for a normal boy (versus a normal girl) is $\mathrm{OR}=1.41$. That means that a normal boy is inclined to read correctly Arabic words 1.41 times more than a normal girl. The significance of that result is that the proportion of correctly read words, out of 100 words, by a normal boy $((\mathrm{Pr})=0.76)$ is higher than the proportion of correctly read word by a normal girl $((\mathrm{Pr})=0.693)$.

- There is a relationship between the gender variable and reading score variable among boys. The cross ratio value for a dyslexic boy versus a normal boy is 0.295 . That means that a dyslexic boy is inclined to read correctly Arabic words 0.295 times than a normal boy. The significance of that result is that that the proportion of correctly read words, out of 100 words, by a dyslexic boy $((\mathrm{Pr})=0.48)$ is lower than the proportion of correctly read word by a normal boy $((\operatorname{Pr})=0.0 .76)$.

- There is a relationship between the dyslexia variable and reading score variable among girls $\left(\chi_{\text {Wald }}^{2}(1)=847.901, p \approx 0<0.05\right)$

The cross ratio value for a dyslexic girl (versus a normal girl) is $\mathrm{OR}=0.146$. This means the dyslexic girl tends to read correctly Arabic words 0.146 times than the tendency of a normal girl. The significance of that result is that the proportion of correctly read words out of a 100 by a dyslexic girl $((\operatorname{Pr})=0.248)$ is lower than the proportion of correctly read words out of a100 by a normal girl. $((\operatorname{Pr})=0.693)$.

In light of the above we can conclude that:

- The achievements of normal boys in the reading test, are the highest, higher than the achievement of the students in all the other groups.

- The achievement of dyslexic girls in the reading test are the lowest, lower than the achievements of the students in all the other groups.

\subsection{Examination of the Relationship between Gender and Dyslexia and Reading Comprehension Score}

The Generalized Linear Implementation Models revealed that at a 0.05 significance level it is possible to conclude that there is a relationship between the interaction gender and dyslexia and the reading comprehension score. For further analysis we examined the relationship between one of the dependent variables and the reading score, given the other independent variable.

The test results at a significance level 0.05 , revealed that:

- In dyslexic students, there is a relationship between the gender variable and the reading comprehension score. The cross ratio value for a dyslexic boy (versus a dyslexic girl) is $\mathrm{OR}=1.643$. That means that a dyslexic boy, in a reading comprehension test, is inclined to answer correctly 1.643 times more than a dyslexic girl. The significance of that result is that the proportion of correct answersfor16 questions of a dyslexic boy ((Pr) $=0.47$ ), is higher than the proportion of correct replies in a reading comprehension test given by a dyslexic $\operatorname{girl}((\operatorname{Pr})=0.32)$. 
- There is no relationship between the gender variable and the reading comprehension score variable among normal students $\left(\chi_{\text {wald }}^{2}(1)=0.106, p=.744>0.05\right.$ ). The proportion of correct answers in the reading comprehension test of normal boys $((\mathrm{Pr})=0.68)$ is nearly identical to the corresponding value for normal girls $((\operatorname{Pr})=0.695)$.

- In boys, there is a relationship between the dyslexia variable and the reading comprehension score variable $\left(\chi_{\text {wald }}^{2}(1)=19.228, p \approx 0<0.05\right)$. The cross ratio value for a dyslexic boy (versus a normal boy) is $\mathrm{OR}=$ 0.556 . That means that in a reading comprehension test a dyslexic boy is inclined to answer correctly 0.566 times of a normal boy inclination to reply correctly. The significance of that result is that the proportion of correct answers for 16 questions, of a dyslexic boy $((\mathrm{Pr})=0.47)$ is lower than the proportion of correct replies in a reading comprehension test given by a normal boy $((\mathrm{Pr})=0.68)$.

- There is a relationship between the dyslexia variable and the reading comprehension score variable among girls $\left(\chi_{\text {Wald }}^{2}(1)=80.303, p \approx 0<0.05\right)$. The cross ratio value for a dyslexic girl (versus a normal girl) is $\mathrm{OR}=$ 0.326 . That means that in a reading comprehension test a dyslexic girls is inclined to answer correctly 0.566 times of a normal girl inclination to reply correctly. The significance of that result is that the proportion of correct answers to 16 questions, of a dyslexic girl $((\mathrm{Pr})=0.32)$ is lower than the proportion of correct replies in a reading comprehension test given by a normal girl $((\operatorname{Pr})=0.695)$.

In summary we can draw the following conclusions:

- The achievements of normal boys and girls in reading comprehension were identical and the highest.

- The achievements of dyslexic girls in reading comprehension were the lowest among the students in all the other groups.

\subsection{Testing the Third Research Hypothesis}

The third hypothesis claims that social competence variables will explain the reading and the reading comprehension scores. To examine this hypothesis, a nonlinear regression was done, using the Generalized Linear Models with a binomially distributed dependent variable with the use of Probit relationship function. The model was applied twice, once for to examine the relationship between social competence variables (dependent variables) and the reading score variable (dependent variable), and once again to examine the relationship between social competence variables (explaining variables) and reading comprehension score variable.

- The empathy explains the reading score $\left(\chi_{\text {Wald }}^{2}(1)=175.631, p \approx 0<0.05\right)$. Obtaining cross ratio, $\mathrm{OR}=2.568$ greater than the value 1 indicates the existence of a positive relationship between empathy and the number of correctly read words, out of 100 words, read by a student.

- The assertiveness explains the reading score $\left(\chi_{\text {wald }}^{2}(1)=69.855, p \approx 0<0.05\right)$. Obtaining cross ratio, $\mathrm{OR}=$ 0.608 smaller than the value 1 , indicates the existence of a negative relationship between assertiveness and the number of correctly read words out of 100 words, read by a student.

- The self-control explains the reading score $\left(\chi_{\text {Wald }}^{2}(1)=10.233, p=.001<0.05\right)$. Obtaining cross ratio, $\mathrm{OR}=$ 1.167 greater than the value1, indicates the existence of a positive relationship between self control and the number of correctly read words out of 100 words, read by a student.

- The cooperation explains reading score $\left(\chi_{\text {wald }}^{2}(1)=30.969, p \approx 0<0.05\right)$. Obtaining cross ratio, $\mathrm{OR}=1.424$ greater than the value 1 indicates the existence of a positive relationship between cooperation and the number of correctly read words out of 100 words, read by a student.

- The empathy explains the reading comprehension score (the number of correct answers to 16 questions given by the student $) \quad\left(\chi_{\text {Wald }}^{2}(1)=13.288, p \approx .00027<0.05\right) \quad$ Obtaining cross ratio, $\mathrm{OR}=1.828$ greater than the value 1 one, indicates the existence of a positive relationship between empathy and the number of correct replies to 16 questions.

- The assertiveness does not explain the reading comprehension score $\left(\chi_{\text {Wald }}^{2}(1)=0.348, p=.555<0.05\right)$

- The self-control explains the reading comprehension score $\left(\chi_{\text {Wald }}^{2}(1)=0.279, p=.597<0.05\right)$.

- The cooperation does not explain the reading comprehension score $\left(\chi_{\text {Wald }}^{2}(1)=0.001, p=.976<0.05\right)$.

\section{Discussion}

The discussion will address each hypothesis separately and at the end a general summarizing discussion will be presented. 


\subsection{Discussion of Hypothesis 1}

The first research hypothesis assumed finding a statistically significant relationship between gender and dyslexia and the emotional-social condition of the student. So that Arab dyslexic adolescent boys and girls, will report on social-emotional characteristics lower than those of Arab normal adolescent boys and girls, and that Arab dyslexic adolescent girls will report of social-emotional characteristics, even lower than those of Arab dyslexic adolescent boys.

In the present study we examined social competence as a social aspect in the first hypothesis, and it was divided into four elements: cooperation, assertiveness, self-control and empathy. The findings in testing that hypothesis indicated that the level of social competence of Arab dyslexic adolescent boys and girls is lower than that of normal Arab adolescent boys and girls, and that the overall social competence level of the Arab dyslexic girls was lower than that of normal girls. These findings confirm the hypothesis that the Arab dyslexic adolescent boys and girls, evaluated their social competence as being low, while Arab dyslexic adolescent girls reported of a very low social competence. However, Arab normal adolescent boys and girls, rated their social competence level as medium to high. These findings are compatible with those of many theoreticians, researchers, and professionals who argue that learning disability has a direct relationship to deficiencies and shortcomings in the social sphere, in children and adults with deficiencies (Yagil, 2006; Lavian Elul, Hertz, \& Lazorovitz, 2003. Margalit, 2014).

Low social competence is being explained as a disability in cognitive processes and difficulties in the processing and understanding of social knowledge effectively, in interpersonal communication and emotional difficulties. As a result, the child finds it difficult to interpret social events efficiently, finds it difficult to focus attention on significant social cues, has difficulty in understanding social situations, draw conclusions and solve problems, analyze wrong environmental information and has trouble understanding others (Margalit, 2014; Neuberger \& Margalit, 1998; Bryan, 1991; Bryan \& Bryan, 1981; Al-Yagon \& Margalit, 2013; Pearl, 1992). The low social competence among children with learning disabilities, reduces their chances to develop normal social relationships and function within them effectively. All of these lead to emotional difficulties. Therefore, these children are described as being frustrated, unmotivated, unstable, rebellious, anxious, depressed and aggressive, have low self-image, poor self-esteem and lonely (Asher \& Gabriel, 1989; Asher et al., 1990; Gresham \& Elliot, 1987; Gresham \& Reschly, 1987). In the current study it was found that the level of social competence of Arab dyslexic adolescent boys and girls, is the lowest. That is consistent with findings in the literature. Ritter (1989) examined the social competence and behavior problems in girls with learning disabilities and presented results that indicated very poorsocial competence, and higher levels of behavior problems when compared with normal adolescents groups (Yagil, 2006; Lavoi, 2005).

Moreover, there is also a significant relationship between the assertiveness variable and the gender and dyslexia variables. The findings suggest that the degree of assertiveness among dyslexic boys and girls is lower than that of normal boys and girls. Dyslexic girls reported a of assertiveness level lower than that of dyslexic boys and the lowest among the entire study population. As for this difference in assertiveness, it should be noted that assertiveness requires self-confidence, a sense of being accepted by peers and the close people, and also a sense of competence and capacity to initiate social meetings, to propose, to ask, and turn to others and expect them to respond positively. Review of the literature shows that students with learning disabilities may have difficulty in these areas which are related to cognitive capacity which affects the social-emotional capacity. That is due to a lack of the adequate socialtraining and verbal skills that affects the capacity to socialize and form social interaction. These difficulties explain the low level of assertiveness and initiative (Margalit, 2014; Heiman, Alunkayi, \& Eden, 2014; Heiman, 2000; Gresham, 1986).

The current study also found that there is a relationship between the gender and dyslexia variable and the cooperation variable. The results indicate that the extent of dyslexic boys and girls cooperation, is lower than the degree of cooperation of normal girls and boys. It also showed that dyslexic girls cooperation is lower than the degree of cooperation of dyslexic boys, while normal boys and girls reported an identical level of cooperationmedium plus. The cooperation variable is very important to adolescents, especially for their popularity in the eyes of their peers. Furthermore, investing in cooperation raises the level of the adolescent's social state and status. As students with learning disabilities have social difficulties, negative self evaluation and low level of assertiveness and empathy, it is logical to assume that the student will have negative feelings about his social success and his interactions, and will then feel rejection and insecurity about his social capacity. In view of these, 
lack of initiative to cooperate is likely. That sort of behavior does not encourage popularity (Ziv \& Ziv, 2001).

Sense of loneliness is defined as a painful emotion that appears when a person is rejected, feels being alienated or misunderstood by others. Thus, the social relationships are not compatible with his social expectations (Heiman, Alunkayi, \& Eden, 2014; Lavian Elul, Raviv, \& run-Lazarowit, 2003; Lev, 2008; Al-Yagon \& Margalit, 2013). Social competence and sense of loneliness are two sides of the same coin (Moin, 1996). Studies showed that the loneliness variable has two main components, emotional and social (Andersson el al., 1987). The findings of the current study confirm this theory, and indicate that Arab dyslexic girls experience a high degree of loneliness most of the time, while, Arab dyslexic boys report feeling lonely only in some situations. Moreover, Arab dyslexic girls report of a sense of loneliness higher than that of normal girls, while normal boys and girls reported that they hardly ever feel lonely. Indeed, the literature suggests that the lack of close and significant social relationships, may lead to a sense of loneliness that is characteristic in adolescence. Epidemiological studies indicated that loneliness is more common in adolescence than in all other age groups (Spector, 2005; Brennan \& Auslander; 1982).

Dyslexic adolescent Arab girls reported of self-image levels lower than those of boys. Another possible explanation is that puberty is characterized by sexual development of boys and girls. Thus, boys and girls behave according to the conventions and the gender functioning set by the Arab culture and society, according to which the boys are less restricted than the girls. During their development, teenage girls experience an inferior status and control on everything they do, therefore their self-esteem is low and consequently their self-is even lower, furthermore, when the society marks them as dyslexic group-an inferior group. (Quatman \& Wastson, 2001).

\subsection{Discussion of Hypothesis 2}

The second research hypothesis assumed finding a statistically significant relationship between gender and dyslexia and the academic level of the student, namely, that the achievements of dyslexic adolescent Arab girls will be lower than the achievements of adolescents dyslexic Arabs boys and the achievement of Arab dyslexic adolescent boys and girls, will be lower than the achievements of normal adolescent boys and girls.

The findings of the reading and reading comprehension tests results, fully confirm the second hypothesis. The study indicated that the abilities and achievements of dyslexic boys and girls are low and limited. They had difficulty to act at the pace of normal students, and their functioning level exhibits a two years delay for their age. That was significantly obvious for dyslexic girls, who in the current study had the lowest achievements.

The research literature that deals with the learning function (Ben-Gal, 2004; Peleg, 2009) claims that the low achievements of adolescents with learning disabilities, are due to their lower capacity at visual memory and auditory-verbal memory processes than normal adolescents. Also, based on self-reporting, they use less memory strategies than normal students. It was also found that adolescents with learning disabilities get over excited during examinations and have more difficulty to regulate their emotions than normal students. Furthermore, students with learning disabilities consider themselves less capable contenders than their normal peers (Lavoi, 2005; Margalit, 2004; Ritter, 1989).

\subsection{Discussion of Hypothesis 3}

The third research hypothesis assumed that the social competence variable will explain the reading and reading comprehension scores, namely, that we will find a positive relationship between social competence factors: empathy, assertiveness, cooperation and self-control and the reading and reading comprehension test scores. The findings show that a positive relationship between three of the four components, empathy, self-control, cooperation and reading test score indeed exists. However, there is a negative relationship between assertiveness and the reading test score. In addition, according to an analysis of the findings indicate that there is a positive relationship between the social competence factors, empathy, cooperation and self-control and the reading comprehension test score. However, there is a negative relationship between assertiveness and the score of this test.

What has been said can explain the current research findings whereby there is a relationship between the four factors of social competence and the reading and reading comprehension test-a relationship between cognitive functioning and social functioning. It turned out that the higher the one variable, the higher is also the other variable-a finding that indicates a positive relationship between them. In other words, if the self-control is high, then the reading and reading comprehension score is high and vice versa. In the current study we show that in dyslexic boys and girls both social competence factors, and academic achievements are low. 


\subsection{General Discussion}

The findings of this study indicate that Arab dyslexic adolescent girls have low level of social competency, high sense of loneliness, low self-image and poor academic achievements in reading and reading comprehension tests. This can be explained on the grounds of cultural perspective. Members of the Joint Committee of Unions in the United States (NJCLD, 2011), highlighted in view of recent research results, and on the basis of scientific neurobiological data, the existence of neurological and neurobiological processes that explain the difficulties emerging in learning disabilities and in the understanding their outcome in various aspects of learning. They emphasized that learning disabilities occur in different groups of students belonging to different cultures, different races, different socioeconomic status in boys and girls. This latest definition displays a dynamic ecological multidimensional model which recognizes the existence of different levels of various disabilities, and combines an observation into social, emotional and cultural factors (Margalit, 2014).

Thus, it is logical to conclude that the Arab culture and its characteristics affect the functioning of female students and even more so of dyslexic girls. In his study, Schwartz (1994) points out the Arab Israeli cultural values: prudence, respect for parents, obedience, family security and hierarchy, rooted in the authority and the power of the culture. The Arab family is patriarchal and has two types of hierarchies: an age hierarchy and a gender hierarchy. Consequently, the Arab society has different educational and cultivation systems, one for girls and women and the other for men and boys (Shulman, 1995). Girls education begins at childhood and includes numerous restrictions and prohibitions. Dyslexic Arab girls are in a complex situation both culturally and socially, as the dyslexia causes the Arab society to appreciate them less than boys because the society has an inborn negative attitudes towards those who are learning disabled. Therefore, the dyslexic girls formulate very low self-identity and self-imagevis-a-vis dyslexic boys or normal girls and boys. They feel rejected and unappreciated, and this takes them into a cycle of isolation, which increases the already existing sense of loneliness. These positions have become part of the social and cultural norms that affect the individual, and particularly the Arab girls.

Crabtree, Zuniga and Ficsher (2010) studied Arab families of children with special needs, and found that their negative attitudes, since most of them are not accepted by society, and the rejection cause a split in the family and non-cooperation between its members in order to circumvent the disability. Usually the blame for the disability is imposed on the mother. The study of Young (1997) which was done in Jordan on families of children with learning disabilities and special needs, indicated the existence of a negative stigma on these families, and only a close knitted family can cope well with the social pressure that sometimes leads to divorcing the mother. Similar findings were obtained in other Arab countries such as Lebanon and Palestine (Atshan, 1997). The results of the current study also support the finding that there is a connection between culture and dyslexia, and that boys and girls suffer from negative attitude, and diminution, which makes them feel inferior to normal boys and girls. Consequently, their social competence level within the Arab society decreases and declines, a situation that increases the sense of loneliness and negatively affects academic functions. Obviously, a student who is emotionally restricted, will never be free academically and this will be reflected in poor scholastic achievement.

To summarize, disabilities have different levels of severity and they vary according to personal characteristics, developmental stages, environmental conditions and cultural affiliation. They have numerous implications on the cognitive-learning area, and on the development of social and emotional functioning. Due to repeated cognitive failures, adolescents with learning disabilities tend to develop emotional stress, low self-image, social competence failure and sense of loneliness. The need to adapt to a social system and acquire social competency which provides high self-image and sense of belonging which reduces the sense of loneliness, requires proper utilization of reading skills (Lamm \& Epstein, 1994).

The current study has several limitations. Some are due to the selection of the study population, the sample and the tools. It would have been worthwhile to examine the perceptions of teachers and parents on the emotional-social-academic aspect of dyslexic students from the Arab sector, in order to get a comprehensive information about the phenomenon and its social, emotional, educational and cultural implications.

It might be worthwhile to expand the research and select a larger sample, broader and more diverse in terms of religion and geographical distribution of residence, to obtain a higher reliability level and credible results.

\section{References}

Adad, M. (2002). Negativism in Adolescence. In Y. W. M. Adadand (Ed.), Crime and Social Deviance: Theory and Practice 
(pp. 50-41). Ramat Gan: Bar-Ilan University.

Bandes-Yaacov, A., \& Friedman, Y. (2000). “Naaleh”-Young Immigrants without Parents. Jerusalem: Henrietta Szold Institute.

Ben-Gal, A. (2004). The Relationship between Self-Concept in the Field of Social Competency, General Anxiety and Social Anxiety among Adolescents with Reading Disability. Master's Degree Thesis. Haifa: Haifa University.

Friedman, A. (2007). Special Children. Hed Hagan, 107-106.

Galantz, Y. (1989). Self-Image Questionnaire. "He (She) So and So ... and I so ...” in Hamad_For a Comprehensive Examination (pp. 199-155). Petach Tikvah Mofet Institute-Barak Information Systems.

Heiman, T. (2000). Social Adjustment of Students with Learning Disabilities. Perspectiva, 16, 42-33.

Heiman, T., Alunkayi, S., \& Eden, S. (2014). Involvement and Victims in the Network among Students with Attention Deficit and Hyperactivity Disorders and without Disorder and the Relationship to Self-Capability, Loneliness and Social Support. Meeting for Social—Educational Work (pp. 112-193). The Education Department the Open University.

Lavian Elul, N. Z., Raviv, S., \& Hertz-Lazarowit, R. (2003). Social Competence Perceptions and Sense of Loneliness of Children with Learning Disability in an Integrated Classroom, and Their Social Behavior Atcooperative and Competitive Physical Activity. Sugiotbechinuchmeyuchadveshikum, 18, 35-19.

Lavian, Z. (1996). Perception of Social Competence and Sense of Loneliness of Students with Learning Disability in the Integrated Classroom and Their Social Behavior in Competitive Physical Activity. Master's Thesis, Haifa: Haifa University.

Lev, D. (2008). To Be Attentive to the Student’s Own Voice-For a Way That Will Allow Him to Opening of the Next Door. Machshavarav-tchumitbachinuchhahumunisty, 3, 67, 65.

Margalit, M. (1994). Learning Disability and Sense of Loneliness. Hed Hagan, 58, 262-258.

Margalit, M. (1996). Development Trends in Special Education: Promotion of Dealing with Loneliness, Friendships and Sense of Coherence. In D. Chen (Ed.), Education, towards the Twenty-First Century (pp. 489-501). Tel Aviv: Ramot.

Margalit, M. (2014). Learning Disabilities: Neuro-Developmental Model after 15 Years, a Meeting Forsocial—Educational Work. 39, 15, 34. Tel-Aviv: Tel-Aviv University.

Margalit, M., \& Tur-Caspa, H. (1998). Learning Disabled: Multidimensional Neuro-Developmental Model. Psychology, 7 , 76-64.

Mazzawi-Maregiya, M. (2001). Staff Attitudes in the Arab Sector towards Integration of Retarded People in the Community. Master's Thesis, Haifa: School of Social Work, Haifa University.

Moss, R. (1988). Theories about Puberty. Tel Aviv: Sifriyat Hapoalim ve hakibutz haartzi.

Neuberger, S., \& Margalit, M. (1998). Coherence Feelings, Loneliness and Social Competence of Students with Special Educational Needs. Megamot, 39, 148-128.

Saada, N. (2009). The Relationship between Self-Image and Motivational Orientation for Studying among Arab Adolescent Students in Israel. Jamaa, 13, 375, 406. Tel Aviv: Sifriyat Poaalim.

Sharonnie, V., \& Bergstein, M. (2010). Loneliness, Self-Image and Social Preference among Adolescents with Learning Disabilities: Self Perceptions versus Teachers Perceptions. Hayoetz Hachinuchi, 16, 114-131.

Shulman, S. (1995). Processes of Maturation and Family Processes-Change and Continuity. In H. Bloom (Ed.), Adolescents in Israel (pp. 60-43). Even Yehuda: Reches.

Spector, N. (2005). Learn from Learning Disabled on a Different Psychology. Tel Aviv: Kriah Betzvaim.

Zahavi, S. (1992). Changing Attitudes of Normal Students towards Disabled Students: A Comparison of Three Methods of Intervention: Bibliotherapy, Values Clarification, Direct Encounter. Haifa: Haifa University.

Ziv, A., \& Ziv, N. (2001). Psychology in Education. Tel Aviv: Yachdav. 


\section{Submit or recommend next manuscript to SCIRP and we will provide best service for you:}

Accepting pre-submission inquiries through Email, Facebook, Linkedin, Twitter, etc A wide selection of journals (inclusive of 9 subjects, more than 200 journals)

Providing a 24-hour high-quality service

User-friendly online submission system

Fair and swift peer-review system

Efficient typesetting and proofreading procedure

Display of the result of downloads and visits, as well as the number of cited articles

Maximum dissemination of your research work

Submit your manuscript at: http://papersubmission.scirp.org/ 